\title{
Nitric oxide and CaMKII: Critical steps in the cardiac contractile response To IGF-1 and swim training
}

\author{
Juan I. Burgos ${ }^{\mathrm{a}}$, Alejandra M. Yeves ${ }^{\mathrm{a}}$, Jorge P. Barrena ${ }^{\mathrm{b}}$, Enrique L. Portiansky ${ }^{\mathrm{c}}$, \\ Martín G. Vila-Petroff ${ }^{a}$, Irene L. Ennis ${ }^{a}{ }^{a}$, \\ a Centro de Investigaciones Cardiovasculares, Facultad de Ciencias Médicas, UNLP-CONICET. Argentina \\ ${ }^{\mathrm{b}}$ Métodos complementarios de diagnóstico, Facultad de Ciencias Veterinarias, Universidad Nacional de La Plata, La Plata, Argentina \\ ${ }^{\mathrm{c}}$ Laboratorio de Análisis de Imágenes, Facultad de Ciencias Veterinarias, Universidad Nacional de La Plata, La Plata, Argentina
}

\section{A R T I C L E I N F O}

\section{Keywords:}

IGF-1, nitric oxide synthase 1

CaMKII

Cardiac contractility

Exercise training

\begin{abstract}
A B S T R A C T
Cardiac adaptation to endurance training includes improved contractility by a non-yet clarified mechanism. Since IGF-1 is the main mediator of the physiological response to exercise, we explored its effect on cardiac contractility and the putative involvement of nitric oxide (NO) and CaMKII in control and swim-trained mice.

IGF-1 increased cardiomyocyte shortening (128.1 $\pm 4.6 \%$ vs. basal; $\mathrm{p}^{<0.05)}$ and accelerated relaxation

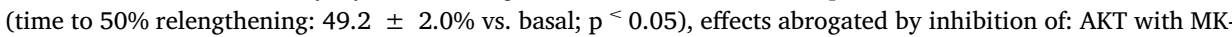
2206, NO production with the NO synthase (NOS) inhibitor L-NAME and the specific NOS1 inhibitor nitroguanidine (NG), and CaMKII with KN-93. In agreement, an increase in NO in response to IGF-1 $(133.8 \pm 2.2 \%)$ was detected and prevented by both L-NAME and NG but not KN-93, suggesting that CaMKII activation was downstream NO. In addition, we determined CaMKII activity (P-CaMKII) and phosphorylation of its target, Thr17-PLN. IGF-1, by a NO-dependent mechanism, significantly increased both (227.2 $\pm 29.4 \%$ and $145.3 \pm 5.4 \%$, respectively) while no changes in the CaMKII phosphorylation site of ryanodine receptor were evident. The improvement in contractility induced by IGF-1 was associated with increased $\mathrm{Ca}^{2+}$ transient $^{2}$ amplitude, rate of decay and SR content. Interestingly, this response was absent in cardiomyocytes from transgenic mice that express a CaMKII inhibitory peptide (AC3-I strain). Moreover, AC3-I mice subjected to swim training did develop physiological cardiac hypertrophy but not the contractile adaptation.

Therefore, we conclude that NO-dependent CaMKII activation plays a critical role in the improvement in contractility induced by IGF-1 and exercise training. Interestingly, this pathway would not contribute to the adaptive hypertrophy.
\end{abstract}

\section{Introduction}

Exercise training is a consolidated approach to reduce cardiovascular risk factors that in the last years has emerged as a promising strategy for the treatment of cardiovascular disease. Several reports have demonstrated its ability not only to positively shift pathologic cardiac remodeling but also to recover abnormal $\mathrm{Ca}^{2+}$ handling and improve contractility $[1,2]$. The first large prospective randomized study to actually provide evidence for a training-induced improvement of ejection fraction and reduction of LV end-diastolic diameter in a mixed population of subjects with ischemic and dilated cardiomyopathy came from Hambrecht et al. [3]. Later, these findings were corroborated by the Exercise in Left Ventricular Dysfunction and Chronic Heart Failure study [4].

Exercise training raises oxygen consumption, and as a consequence cardiac output and oxygen transport increase to match the metabolic demand. Moreover, there is a cardiac adaptation to aerobic exercise training that includes cardiomyocyte hypertrophy and improved contractility, as the most important events. This response is mediated by the production and release of several growth factors and other humoral mediators among which, the insulin-like growth factor 1 (IGF-1) seems to be the most relevant. IGF-1 increases in response to exercise training both in experimental animal models and in athletes compared with healthy sedentary controls $[5,6]$. Furthermore, IGF-1 has been demonstrated to be produced in cardiovascular tissue, where it likely exerts autocrine/paracrine effects. Indeed, IGF-1 binding to its specific receptor (IGF-1R) stimulates, at least, two canonical pathways in cardiomyocytes: the PI3K/AKT and the ERK cascade [7].

The key role played by IGF-1 in the exercise-induced hypertrophic response has been convincingly demonstrated by several research

\footnotetext{
* Corresponding author at: Centro de Investigaciones Cardiovasculares, Facultad de Ciencias Médicas, UNLP, Calle 60 y 120 , 1900 La Plata, Argentina.

E-mail address: iennis@med.unlp.edu.ar (I.L. Ennis).
} 
groups using different experimental models. Transgenic mice with cardiomyocyte-specific knockout of the IGF-1 receptor (IGF-1R) failed to develop cardiac hypertrophy in response to 5 weeks of swim training [8]. Moreover, inhibition or genetic ablation of PI3K or AKT1 prevents physiological cardiac hypertrophy, supporting a key role for this signaling pathway in stimulating cardiomyocyte growth in response to physical exercise [9-12].

With respect to the cardiac positive inotropic response to exercise training, the underling subcellular mechanism has not been completely clarified yet. Importantly, there is still contradictory data regarding the inotropic consequences of IGF-1 in experimental animal models of cardiomyopathies [13-15]. The PI3K/AKT [16] pathway, different isoforms of the nitric oxide synthases (NOS) [16-18] and/or the activation or inhibition of the $\mathrm{Ca}^{2+} /$ calmodulin-dependent kinase II (CaMKII) $[19,20]$ are among the most relevant signaling cascades proposed to be responsible for the improvement in $\mathrm{Ca}^{2+}$ handling that leads to the enhanced contractility associated with exercise training. In addition, Kemi et al. $[19,21]$ reported that aerobic interval training increases the expression of the sarcoplasmic reticulum $\mathrm{Ca}^{2+}$ pump (SERCA2a), significantly improving myocardial $\mathrm{Ca}^{2+}$ handling. Similar results were also reported in mouse and dog models of heart failure subjected to exercise training [22,23]. Moreover, Kim et al. proposed that chronic treatment with IGF-1 enhances myocyte contraction and relaxation functions due to an AKT-dependent up-regulation of SERCA2a in isolated rat cardiomyocytes [24]. On the other hand, there is strong evidence supporting a key role played by NO in the positive inotropic response to exercise [17]. The PI3K/AKT pathway has, as one of its downstream molecular targets, the NO synthases (NOS) that once phosphorylated increase NO production [25-27]. In this regard, Ziolo's group showed in isolated cardiomyocytes from wild type mice that exercise training increases contraction and improves relaxation in an NOS1 dependent manner and that the exercise-induced increase in cardiac function was prevented in trained mice with cardiomyocyte specific deletion of NOS1 (NOS1KO) [17,18]. They proposed as the molecular mechanism responsible for the enhanced $\mathrm{Ca}^{2+}$ cycling a NOS1-mediated increase in the phosphorylation of phospholamban (PLN) at the residue serine 16 (Ser16) probably due to inhibition of a phosphatase, leading to an increase in sarcoplasmic reticulum (SR) $\mathrm{Ca}^{2+}$ content that results in an enhancement in $\mathrm{SR}-\mathrm{Ca}^{2+}$ release $[17,18]$. However, the connection between NO and the phosphatase is only speculative at present. At this point it, is interesting to remember that PLN is also phosphorylated by CaMKII, and this kinase can be activated not only by an increase in intracellular $\mathrm{Ca}^{2+}$ but also by NO $[27,28]$. Interestingly, CaMKII activation has been recently proposed to be involved in the cardiac contractile adaptation to exercise training [19].

The aim of the present work was to investigate in mice the cardiac contractile response to a swim routine and IGF-1 stimulation, specifically focusing on the role played by CaMKII and NO and the putative relationship between both. We hypothesize that a NO-dependent activation of CaMKII is involved in the contractile cardiac adaptation to exercise training.

\section{Materials and methods}

All procedures followed during this investigation conform to the Guide for the Care and Use of Laboratory Animals published by the US National Institutes of Health Eighth Edition (National Academies Press, 2011) and the experimental protocol was approved by the Animal Welfare Committee of La Plata School of Medicine.

Wild-type (WT) C57BL/6 mice (WT, Jackson Laboratories, Bar Harbor, ME, USA) (18 to $20 \mathrm{~g}$ ) were used as controls. Transgenic mice, generated in the C57BL/ 6 background, with cardiomyocyte-delimited transgenic expression of either a CaMKII inhibitory peptide (AC3-I) or a scrambled control peptide (AC3-C) were used [29]. We chose the transgenic mice model instead of a shRNA knock down model because we were interested in exploring the cardiac consequences of a chronic intervention (6 weeks swim routine) and the efficiency of cardiomyocyte transduction (i.e. shRNA) is variable and not homogeneous, usually requiring repeated injections for sustained long term expression $[30,31]$. Even though disparity exists between sexes in cardiovascular physiology and disease that importantly include differential activation and modification of myocardial CaMKII [32,33]; we conducted this study only in male mice in order to minimize the amount of variation in the results. Animals, studied at the age of 8-10 weeks, were kept at constant temperature $\left(23^{\circ} \mathrm{C}\right)$ in a standard light/dark cycle $(12: 12 \mathrm{~h})$, fed ad libitum and had free access to water.

\subsection{Cardiomyocyte isolation}

Male C57BL/6 and transgenic mice were sacrificed by cervical dislocation and hearts were rapidly excised. Cardiac myocytes were isolated by collagenase-based enzymatic digestion according to a technique previously described [34]. After isolation cells were kept in a HEPES solution, containing (in mmol/L): $146.2 \mathrm{NaCl}, 4.7 \mathrm{KCl}, 1.8$ $\mathrm{CaCl}_{2}, 10.0$ HEPES, $0.4 \mathrm{NaH}_{2} \mathrm{PO}_{4}, 1.1 \mathrm{MgCl}_{2}, 10$ glucose ( $\mathrm{pH}$ adjusted to 7.4 with $\mathrm{NaOH})$, at room temperature $\left(20-24{ }^{\circ} \mathrm{C}\right)$ until use.

\subsection{Calcium transient and cell shortening measurements}

$\mathrm{Ca}^{2+}$ transient and cardiomyocyte shortening measurements were performed at room temperature, as previously described [15]. Briefly, the cardiomyocytes were placed in a perfusion chamber on the stage of an inverted microscope (Nikon), continuously superfused with a HEPES solution and stimulated via 2-platinum electrodes on either side of the bath at $0.5 \mathrm{~Hz}$. Resting cell length and cell shortening were measured using a video-based motion detector (Ionoptix) and stored by software for an off-line analysis. The average values of the inotropic response were calculated after 5,10 or $15 \mathrm{~min}$ of incubation with IGF-1 or no drug (control). The time to $50 \%$ relengthening was measured to evaluate the effect of IGF-1 or chronic training on relaxation.

For intracellular $\mathrm{Ca}^{2+}$ measurements isolated cardiomyocytes were loaded with $10 \mu \mathrm{mol} / \mathrm{L}$ Fura-2 AM (Molecular probes) during $12 \mathrm{~min}$ at room temperature. The ratio of the Fura- 2 fluorescence $(510 \mathrm{~nm})$ obtained after exciting the dye at 340 and $380 \mathrm{~nm}$ was taken as an index of free cytosolic $\mathrm{Ca}^{2+}$. Time to $50 \%$ relaxation was used as a parameter of the rate of $\mathrm{Ca}^{2+}$ decay. For SR $\mathrm{Ca}^{2+}$ content measurement, a solution containing $10 \mathrm{mmol} / \mathrm{L}$ caffeine was rapidly applied onto cells. The amplitude of the caffeine-induced $\mathrm{Ca}^{2+}$ transient was used to estimate sarcoplasmic-reticulum $\mathrm{Ca}^{2+}$ content. The pharmacologic inhibitors, when corresponding, were preincubated $10 \mathrm{~min}$ before IGF-1.

\subsection{Measurement of intracellular nitric oxide production in cardiac myocytes}

Cardiac myocytes were loaded with $5 \mu \mathrm{mol} / \mathrm{L}$ DAF-FM diacetate (Molecular probes) for $30 \mathrm{~min}$ at room temperature, and imaged by epifluorescence on a Zeiss 410 inverted confocal microscope (LSM Tech, Pennsylvania, USA). Excitation at $488 \mathrm{~nm}$ was provided by an argon laser and emission was collected in a range of $510-530 \mathrm{~nm}$ as previously described [35]. Photographs were taken every minute, during $18 \mathrm{~min}$. in the absence of any drug (control) or stimulated with IGF-1 $10 \mathrm{nmol} / \mathrm{L}$. The inhibitors, when indicated, were preincubated 10 min before IGF-1. Image $\mathrm{J}$ software was used for the analysis. Results were expressed as a percentage of time zero.

\subsection{Western blot analysis}

Mouse hearts were perfused for $15 \mathrm{~min}$ on a Langendorff apparatus in the presence or absence of $10 \mathrm{nmol} / \mathrm{L} \mathrm{IGF-1}$ with/without different pharmacologic inhibitors and subsequently homogenized. Equal amounts of proteins were seeded in a $10 \%$ SDS polyacrylamide gel and 
A
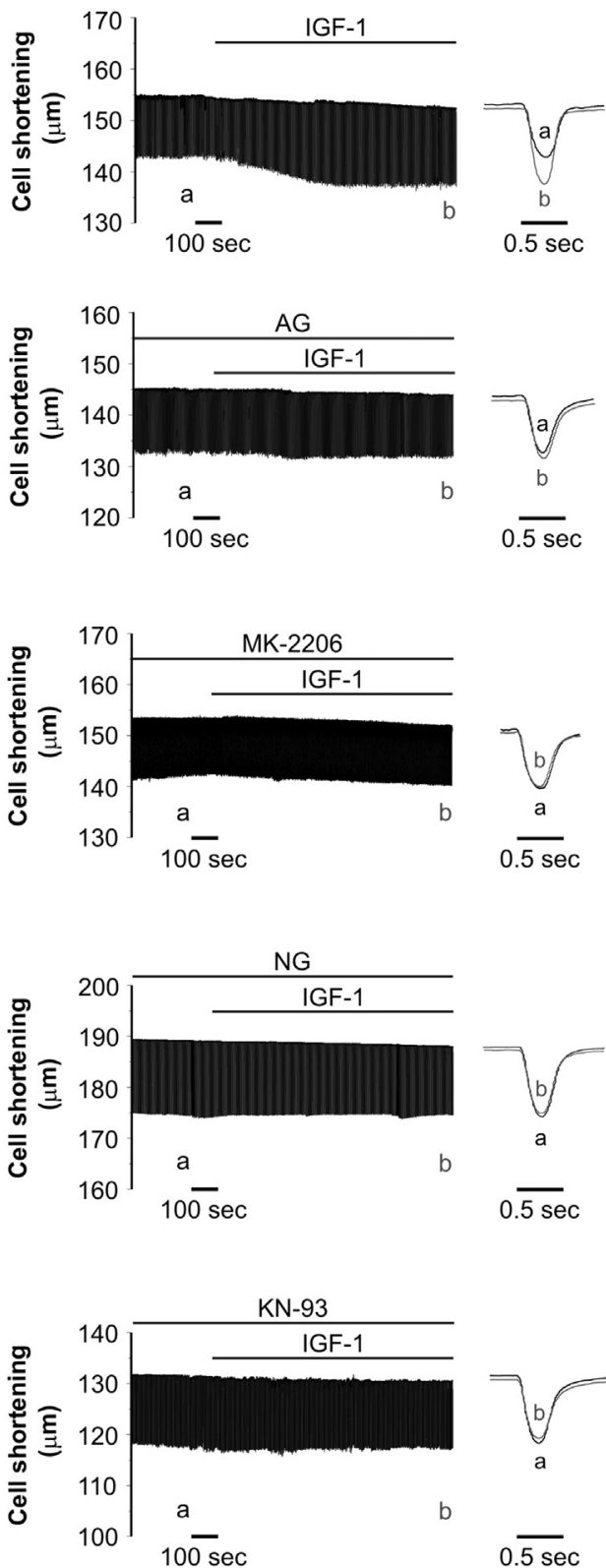

B
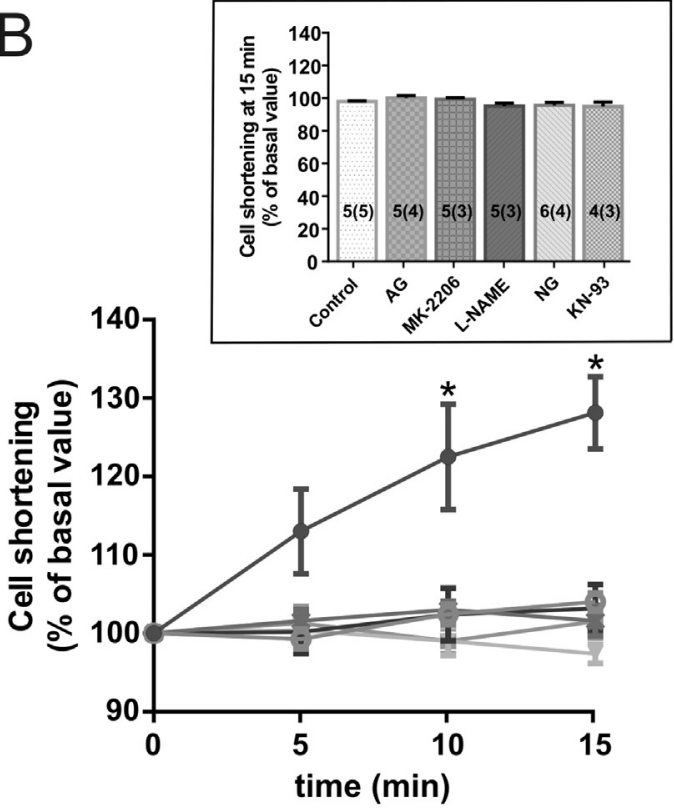

$\rightarrow$ IGF-1 $(n=8 ; N=7) \quad \rightarrow$ IGF-1+L-NAME $(n=5 ; N=4)$

- $\quad$ IGF-1+AG $(n=7 ; N=5) \quad-\quad$ IGF-1+NG $(n=5 ; N=4)$

* IGF-1+MK-2206 (n=5;N=3) $=$ IGF-1+KN-93 (n=6;N=5)

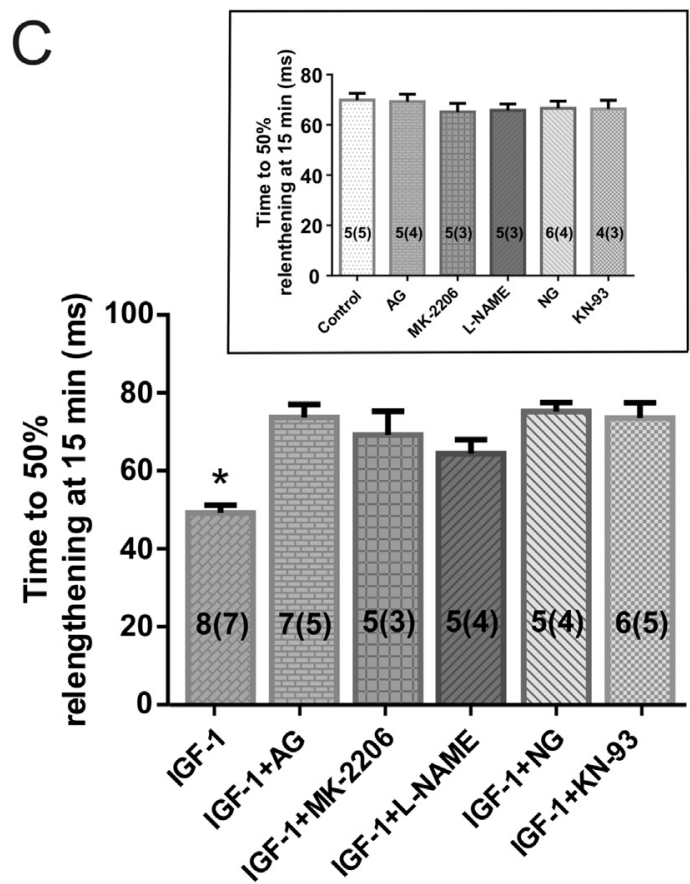

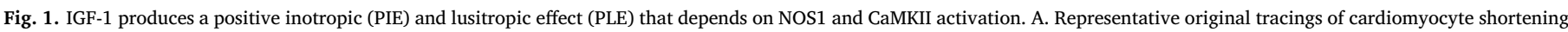

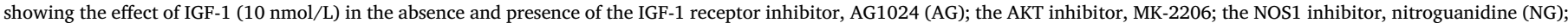

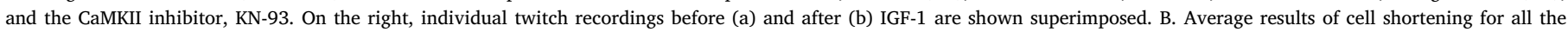

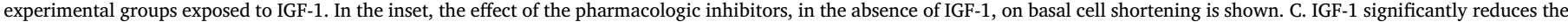

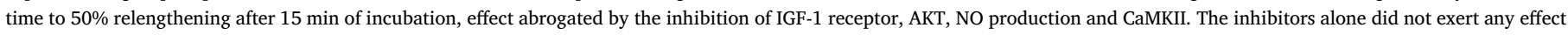

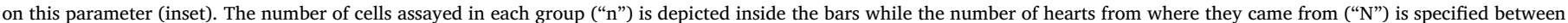
parentheses. * indicates p $<0.05$ vs. control, Kruskal Wallis 1-way ANOVA and Dunn's Method.

transferred to polyvinylidene difluoride membranes. Blots were probed overnight with the antibodies raised against phospho-Ser473 protein kinase B (P-AKT, 1:1000; Cell Signaling), neuronal NO synthase (NOS1, 1:1000; Santa Cruz Biotechnology) phospho-Ser1417-NOS1 (P-NOS1, 1:2000; Abcam), phospho-Thr286-CaMKII (P-CaMKII, 1:1000; Abcam), phospho-Thr17-PLN (2:500; Badrilla), phospho-Ser16-PLN (2:500;
Millipore) and phospho-Ser2814-RyR2 (1:5000; Badrilla). GAPDH detection (1:10,000; Santa Cruz Biotechnology) was used as loading control. Immunoreactivity was visualized by a peroxidase-based chemiluminescence detection kit (Immobilon Western Millipore) using a Chemidoc Imaging System. The signal intensity of the bands in the immunoblots was quantified by densitometry using Image $\mathrm{J}$ software 
A

DAF-FM Fluorescence
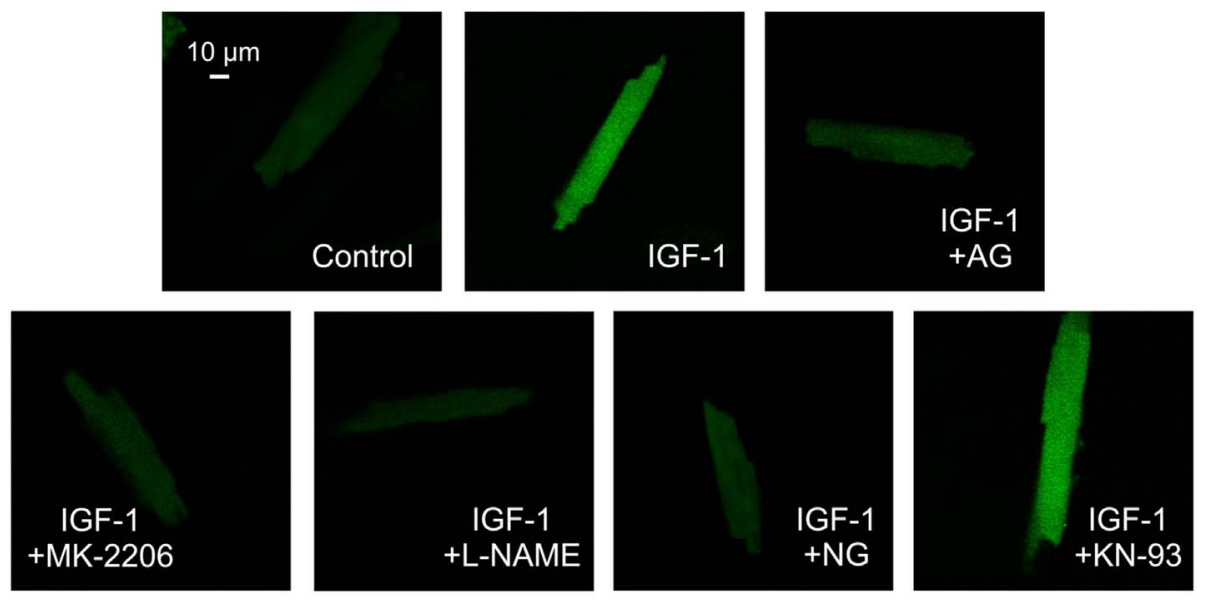

Fig. 2. IGF-1 stimulates cardiomyocyte NOS1-dependent NO production. A. Representative fluorescent images of cardiomyocytes loaded with the NO sensitive fluorescent dye DAF-FM and exposed to IGF-1 in the absence or presence of different inhibitors. B. Average results of DAF-FM fluorescence during $15 \mathrm{~min}$ of incubation with IGF-1 for the different experimental groups. The inhibition of CaMKII with KN-93 did not alter IGF-1-induced NO production. None of the pharmacologic inhibitors used exert an effect on cardiomyocyte NO production in the absence of IGF-1 (inset). The number of cells assayed in each group ("n") is depicted inside the bars while the number of hearts from where they came from ("N") is specified between parentheses. * indicates $\mathrm{p}<0.05$ vs. control at the same time points, Kruskal Wallis 1-way ANOVA and Dunn's Method.

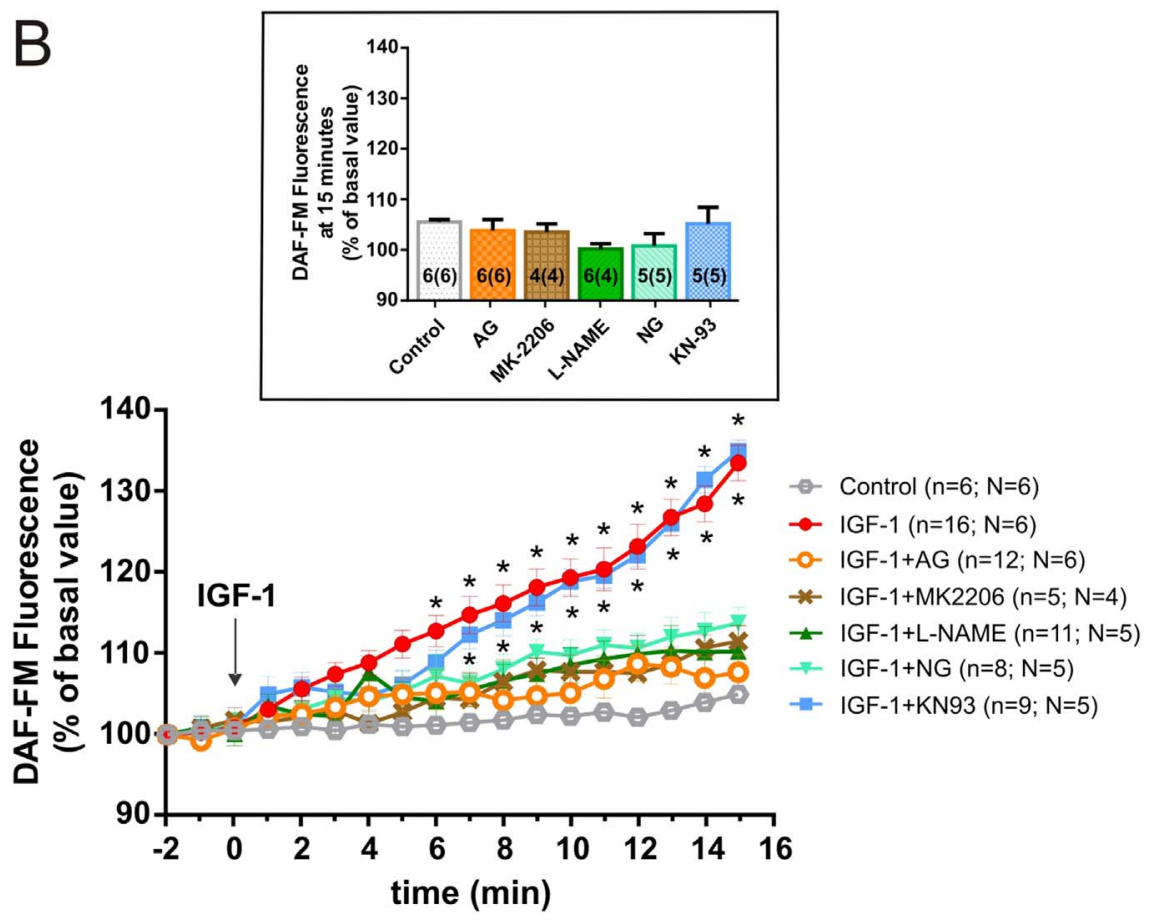

(NIH, USA).

\subsection{Murine exercise protocol}

Mice were randomly assigned to a sedentary (Sed) or swim-trained (Swim) group and divided in six groups: (1) C57 Sed, (2) C57 Swim (3) AC3-C Sed, (4) AC3-C Swim, (5) AC3-I Sed and (6) AC3-I Swim. The swim training protocol was modified from a previously published procedure [36]. Briefly, adult mice in the Swim group were trained, free-of-loading, 5 days/week, twice a day for 6 weeks in warm water $\left(30-32{ }^{\circ} \mathrm{C}\right)$. The first week consisted of a training adaptation period; starting with $20 \mathrm{~min} /$ day of swimming and increased daily by $20 \mathrm{~min}$ up to $90 \mathrm{~min}$. Mice in the Sed group were placed in the swimming tank for $10 \mathrm{~min}$ twice a week to mimic the water stress associated with the experimental protocol. After the final swim session mice were overnight fasted, then weighed and sacrificed. Hearts were washed with PBS, drained by gentle squeeze using cotton gauze and weighed. Right tibias were gently excised and all muscle was removed in order to expose the whole bone for precise measurements with caliper rule. Heart weight (HW)/tibia length (TL) was calculated.

\subsection{Echocardiographic evaluation}

Echocardiagraphy was performed with the Vevo 770 coupled to a $30 \mathrm{MHz}$ transducer (Visual Sonics). Images were acquired in M-mode on the left ventricular muscle thickness and analyzed by a blinded investigator.

\subsection{Histological analysis studies}

Ventricular tissue was fixed in buffered $10 \%$ formaldehyde and paraffin-embedded. LV coronal sections ( $4 \mu \mathrm{m}$ thick) at the equator were stained with hematoxilin-eosin or picrosirius red (Direct Red 80 Aldrich) for blinded determination of cardiomyocyte cross-sectional area (CSA) and quantitation of fibrosis (\% of interstitial collagen). CSA was calculated in a minimum of 50 cardiomyocytes per experimental 
A

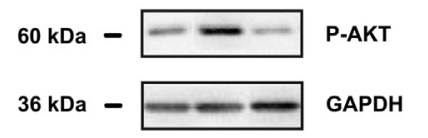

B

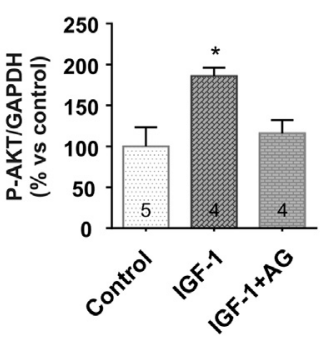

C
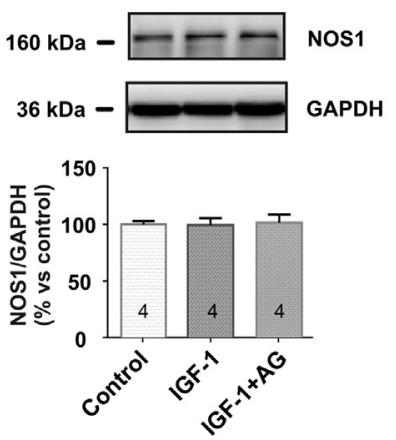
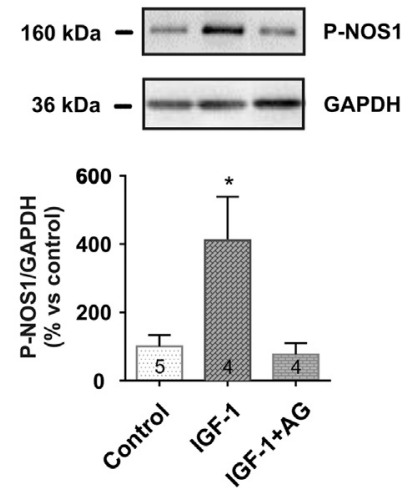

$\mathrm{D}$

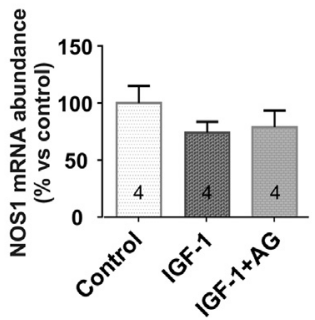

Fig. 3. IGF-1 increases AKT and NOS1 activity. Typical western blots and average data showing that IGF-1 induces both AKT (A) and NOS1 (B) phosphorylation while not increasing NOS1 protein expression (C) through an IGF-1 receptor-dependent mechanism. D. NOS1 mRNA abundance was quantified by real time RT-PCR demonstrating that IGF-1 does not up-regulate this enzyme under acute exposure. The number of experiments (n) in each group is depicted inside the bars. * indicates $\mathrm{p}<0.05$ vs. all other groups, Kruskal Wallis 1-way ANOVA and Dunn's Method.

condition from at least 4 different mice after digitalization at $200 \times$ magnification in an image analysis system (Image-Pro Plus software). The investigator was unaware of the treatment groups and codes were disclosed after statistical analyses.

\subsection{Real time RT-PCR}

Brain natriuretic peptide (BNP) and NOS1 mRNA expression were assessed by real-time RT-PCR and normalized to GAPDH following the procedure described previously [1]. Briefly, total RNA was isolated from cardiac tissue using Trizol ${ }^{\mathrm{TM}}$ Reagent (Invitrogen). RNA $(0.8 \mu \mathrm{g})$ was reverse transcribed using the MMLV (Invitrogen). A dilution of the resulting cDNA was used to quantify the relative content of mRNA by real-time PCR (iCycler iQ real-time PCR detection system, Bio-Rad) using appropriate primers and SYBR Green as the fluorescent probe. The following primers, designed using Primer3 software, were used: $5^{\prime}$ GCTGTAACGCACTGAAGTTGT-3' (forward) and 5'-TCAAAGGTGGTCCCAGAGCT-3' (reverse) for BNP; 5'-CAACAGCGTCTCCTATTC-3' (forward) and 5'-CGAAGACTGAGAACCTCACATT-3' (reverse) for NOS-1; 5'-CATGGCCTTCCGTGTTCCTA- $3^{\prime}$ (forward) and 5'-TGCTTCACCACCTTCTTGATG-3' (reverse) for GAPDH.

\subsection{Chemicals}

All drugs used in the present study were analytical reagents. IGF-1: recombinant mouse insulin like growth factor $-1(10 \mathrm{nmol} / \mathrm{L}$, Cat \# PMG0075 from Gibco by Life Technologies); AG-1024 (100 nmol/L Cat \# sc-205,907 from Santa Cruz Biotechnology); AKT inhibitor MK-2206

(500 nmol/mL Cat \# S1078 from Selleck Chemicals); KN-93 (2.5 $\mu \mathrm{mol} /$ L Cat 422,708 from Calbiochem); nitroguanidine (NG $240 \mathrm{nmol} / \mathrm{L}$, Cat 490,070 from Calbiochem), nitro-l-arginine methyl ester (L-NAME $2.5 \mathrm{mmol} / \mathrm{L}$, Cat N5751 from Sigma). KN-93, MK-2206 and AG-1024 were diluted in DMSO (final concentration $\leq 0.01 \%$ ).

\subsection{Statistical analysis}

Results are expressed as mean \pm SEM. The non-parametric Wilcoxon rank-sum test and Kruskal-Wallis 1-way ANOVA, Dunn's Method were used for statistical comparisons when appropriate. Differences were considered significant at $\mathrm{p}^{<0.05}$. All analyses were made using GraphPad Prism 5.0 (GraphPad Software, USA).

\section{Results}

\subsection{IGF-1 improves cardiomyocyte contractility}

Cardiac contractility was explored by measuring cell shortening in isolated cardiomyocytes. IGF-1 (10 nmol/L) acutely increased cardiomyocyte shortening, reaching its peak at $\sim 15 \mathrm{~min}$ of incubation as it can be appreciated in the typical recordings and overall results shown in Fig. 1A-B. This positive inotropic effect was associated with an acceleration of cardiomyocyte relaxation as revealed by the significant reduction in the time to $50 \%$ relengthening (Fig. 1C). As expected, both effects were abrogated by the inhibition of the IGF-1 receptor (IGF-1R) with AG-1024 (100 nmol/L) and its downstream kinase AKT with MK$2206(500 \mathrm{nmol} / \mathrm{L})$. To get further insight into the subcellular mechanism involved, we examined cell shortening after inhibition of the NOS that are well recognized targets of AKT. We used L-NAME ( $2.5 \mathrm{mmol} / \mathrm{L})$, a non-isoform specific NOS inhibitor, as well as nitroguanidine (NG, $240 \mathrm{nmol} / \mathrm{L}$ ) a specific inhibitor of the neuronal isoform of NOS (NOS1). Under these experimental conditions, the positive inotropic and lusitropic response to IGF-1 were significantly attenuated. Similar results were obtained when CaMKII, a putative target of NO, was inhibited using KN-93 $(2.5 \mu \mathrm{mol} / \mathrm{L})$. None of these inhibitors exerted an effect on contractility or the time to $50 \%$ relengthening when added in the absence of IGF-1 (insets of panels B and C, respectively). Control cells not exposed to IGF-1, showed no significant changes in contractility during the same experimental period as those exposed to IGF-1.

\subsection{IGF-1 stimulates cardiomyocyte NO production}

To confirm the participation of NO in the IGF-1-induced inotropic response we evaluated its production by epifluorescence in isolated cardiomyocytes exposed to IGF-1. IGF-1 increased the NO-dependent fluorescent signal, effect that was significantly attenuated by the blockade of IGF-1R, AKT and NOS1 (Fig. 2). Interestingly, the inhibition of CaMKII did not alter IGF-1 induced NO production, suggesting that, if they were both components of the same signaling pathway, CaMKII should be downstream of NO. This data was additionally supported by immunoblot determinations which confirmed that IGF-1 induced both AKT and NOS1 phosphorylation through an IGF-1R dependent mechanism (Fig. 3A-B). We excluded the possibility that an increase transcription of NOS1 was collaborating in the augmented production of NO by quantifying NOS1 protein and mRNA abundance (Fig. 3C-D).

\subsection{IGF-1 and intracellular $\mathrm{Ca}^{2+}$ handling}

In order to gain further understanding of the signaling cascade underlying the positive inotropic/lusitropic effect of IGF-1, we evaluated CaMKII activation by determining its autophosphorylation and the phosphorylation of its downstream targets, residue threonine 17 (Thr17) of PLN and serine 2814 of the ryanodine receptor (Ser2814RyR2). IGF-1 significantly increased the phosphorylation of CaMKII and 
A
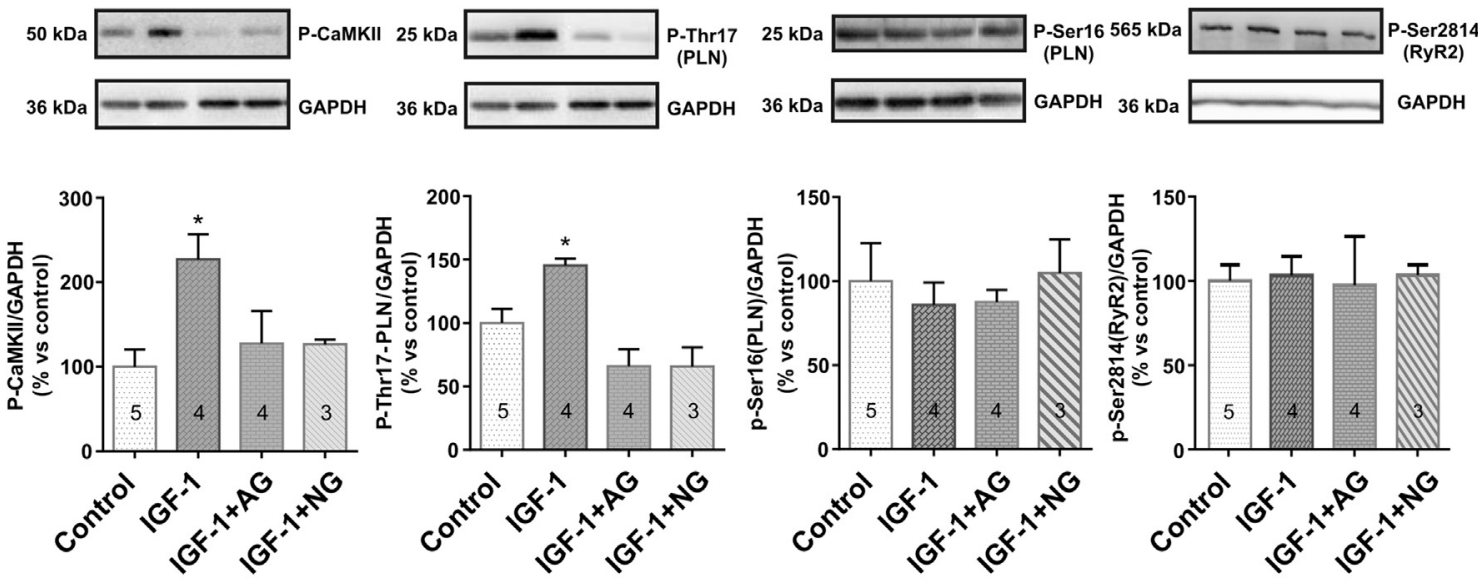

B
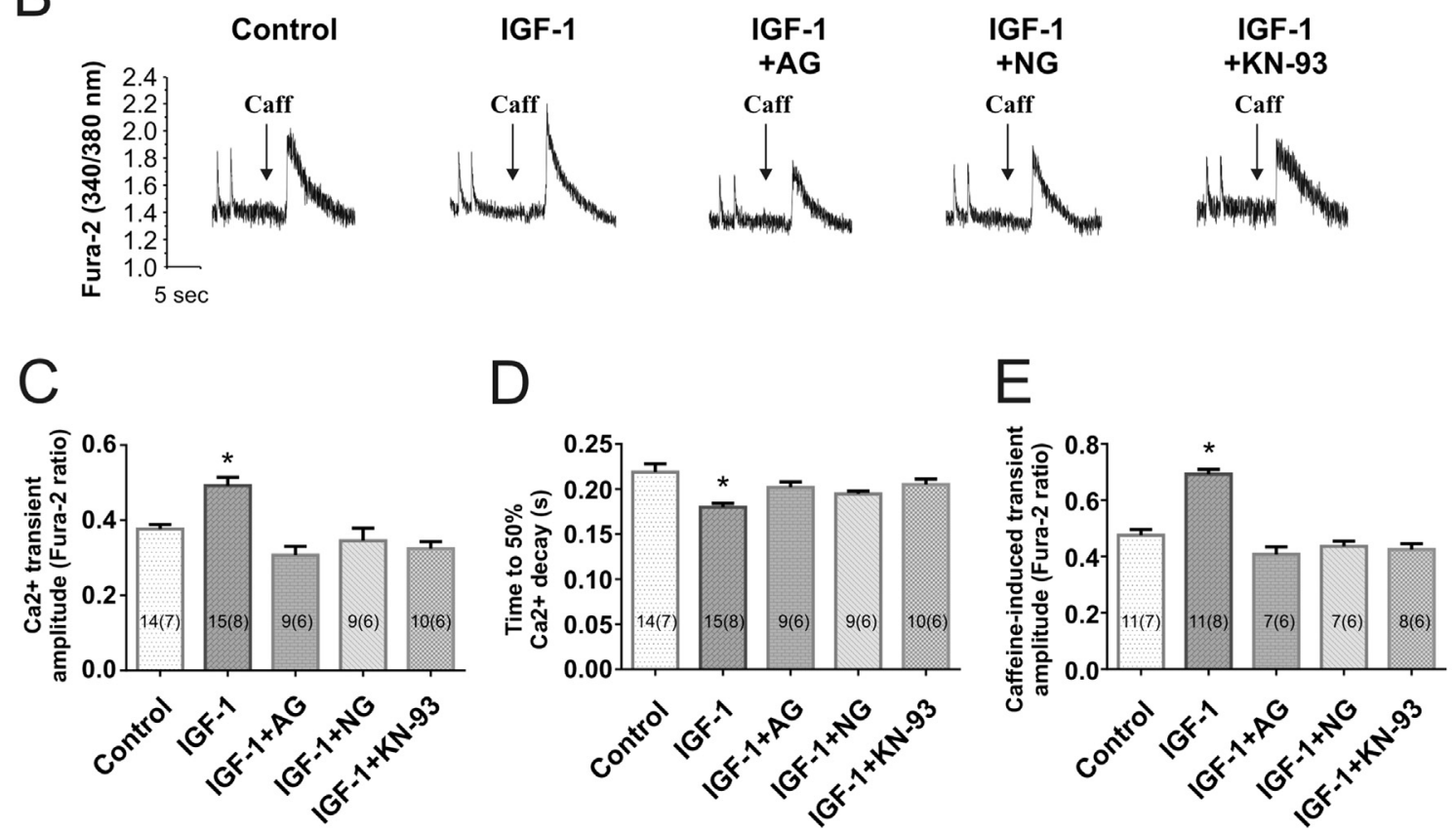

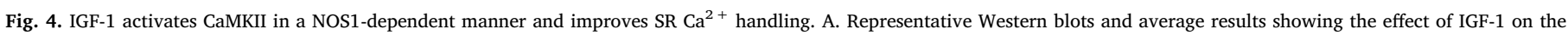

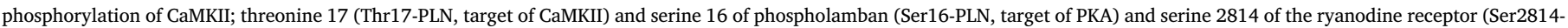

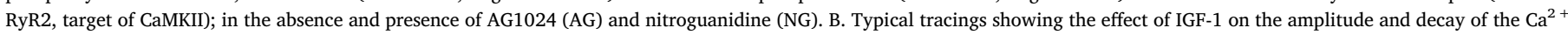

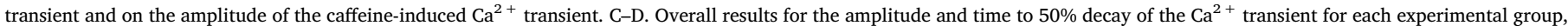

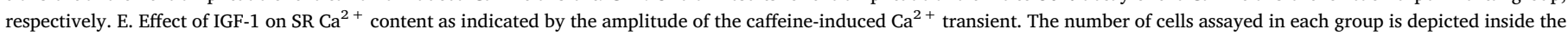

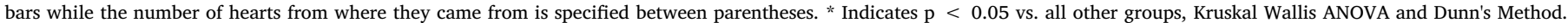

Thr17-PLN, effects that were cancelled not only by the blockade of IGF$1 \mathrm{R}$ with AG but also by the inhibition of NOS1. On the contrary, no significant differences were detected in the phosphorylation of either the CaMKII site on RyR2 (Ser2814) or the PKA site on PLN (Ser16) (Fig. 4A). These results are in line with those of Fig. 2, suggesting that NO production and CaMKII activation are steps of the same intracellular signaling cascade, being CaMKII downstream of the increase in NO. Since PLN phosphorylation is expected to increase the activity of the SR $\mathrm{Ca}^{2+}$ pump (SERCA2a) augmenting SR $\mathrm{Ca}^{2+}$ content, we analyzed the amplitude and decay of $\mathrm{Ca}^{2+}$ transients in isolated cardiomyocytes exposed to IGF-1. We found that IGF-1 induced a significant increase in the amplitude of the $\mathrm{Ca}^{2+}$ transient while reducing its time to $50 \%$ decay (Fig. 4B-D). Moreover, IGF-1 significantly increased the $\mathrm{SR} \mathrm{Ca}^{2+}$ content as revealed by the greater amplitude of the caffeine- induced $\mathrm{Ca}^{2+}$ transient (Fig. 4E).

\subsection{IGF-1 on cardiomyocyte contractility in a transgenic mice model}

To confirm the participation of CaMKII in the inotropic/lusitropic effects of IGF-1 we performed another set of experiments in transgenic mice that express an inhibitory peptide of the kinase (AC3-I strain, and their corresponding control AC3-C). As shown in Fig. 5, IGF-1 failed to increase cell shortening and accelerate relaxation in isolated cardiomyocytes from AC3-I mice, while this effect was preserved in AC3-C mice. 

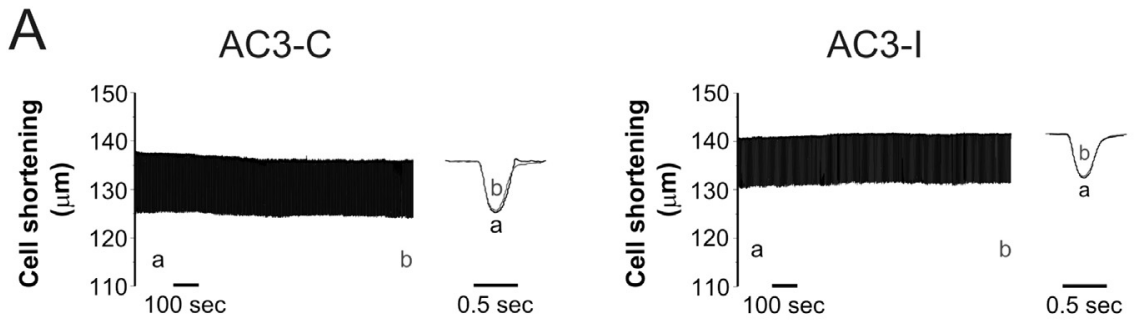

Fig. 5. IGF-1 fails to affect cardiomyocyte contractility in transgenic AC3-I mice. Typical tracings (A) and overall results (B) showing that the positive inotropic effect of IGF-1 was present in isolated cardiomyocytes from control transgenic AC3-C mice and absent in myocytes that express the CaMKII inhibitory peptide (AC3-I strain). The number of cells assayed in each group is depicted inside the bars while the number of hearts from where they came from is specified between parentheses. * Indicates $\mathrm{p}<0.05$ vs. all other groups, Kruskal Wallis ANOVA and Dunn's Method.
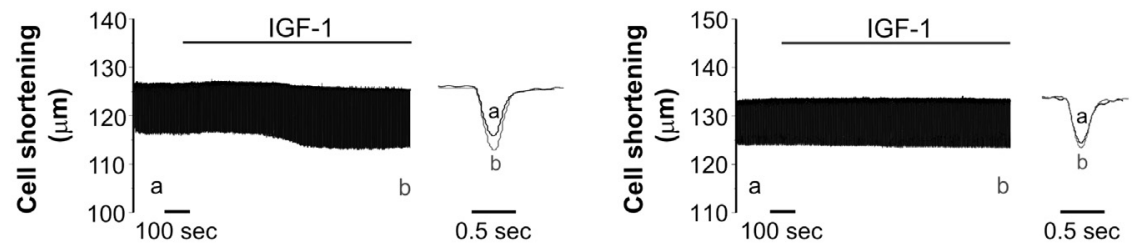

$\mathrm{B}$
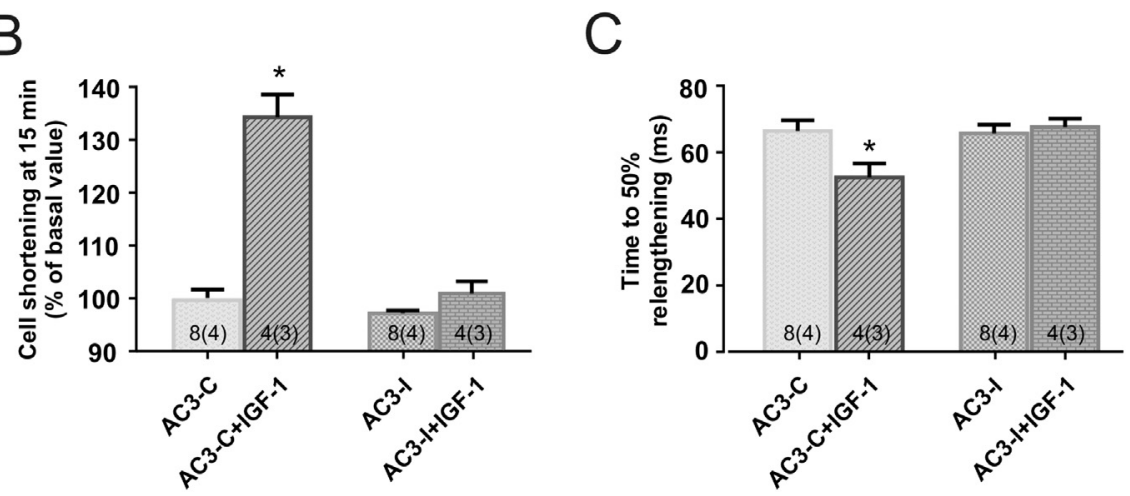

\subsection{Cardiac adaptation to swim training in wild type and transgenic mice}

Having demonstrated the key role played by CaMKII in the positive inotropic/lusitropic effect of IGF-1 in cardiac myocytes we further explored its role in the setting of hearts with physiological cardiac hypertrophy. In pursuing this aim, we subjected mice to 6 weeks of exercise training by swimming. At the end of this period, physiological cardiac hypertrophy was evidenced by the increase in cardiac mass and cross sectional area of cardiomyocytes without the presence of fibrosis. The physiological nature of hypertrophy was reinforced by the absence of up-regulation of brain natriuretic peptide, a molecular marker of pathological hypertrophy. Physiological cardiac hypertrophy was accompanied by an increase in fractional shortening detected by echocardiography (Fig. 6A-F). In homogenates from these hearts we determined the phosphorylation state of AKT, CaMKII, Thr17-PLN and Ser2814-RyR2. In the hypertrophied hearts, we found an increased phosphorylation in AKT, CaMKII and Thr17-PLN but not in Ser2814RyR2, in agreement with the results obtained with IGF-1 stimulation (Fig. 6G).

Transgenic AC3-I and AC3-C mice, were subjected to the same swim protocol, and even though AC3-I myocytes had not shown the positive inotropic response to IGF-1 (see Fig. 5), they did develop physiological cardiac hypertrophy (Fig. 7A-D). As expected, only in the AC3-C mice the swim routine induced CaMKII activation increasing P-Thr17-PLN. Again, no significant changes in the phosphorylation status of Ser16PLN or Ser2814-RyR2 were detected (Fig. 7E). In agreement with these biochemical modifications an increase in cardiac contractility and acceleration of relaxation were detected exclusively in the AC3-C trained mice (Fig. 7F-H). As a whole, these results support that CaMKII is not involved in the hypertrophic response although it is critical for the adaptive contractile response to exercise training.

\section{Discussion}

The results presented herein provide strong evidence that CaMKII plays an essential role in the positive inotropic/lusitropic response to IGF-1 and in the adaptive improvement in contractility associated to exercise training. Taken together our findings allow us to propose that exercise training, through the release of IGF-1, induces the phosphorylation of AKT which in turn activates NOS1 increasing myocardial NO production. The increase in NO would be responsible for CaMKII activation improving $\mathrm{SR} \mathrm{Ca}^{2+}$ handling and therefore contractility. The underling mechanism would involve the phosphorylation of Thr17-PLN increasing SERCA2a activity. Interestingly, the experiments performed in AC3-I/AC3-C transgenic mice also revealed that CaMKII, although critically involved in the inotropic/lusitropic response to IGF-1 and swim training, is not required for the development of physiological cardiac hypertrophy.

In this work, we specifically investigated the role played by the NOCaMKII axis in the inotropic response to IGF-1, the major mediator of the cardiac adaptation to exercise training. NO is a well-established regulator of cardiac contractility. Both NOS1 and NOS3 -the endothelial isoform- are constitutively expressed in cardiomyocytes; however, while NOS1 is localized to the SR, NOS3 is localized to the caveolae, mediating independent, and in some cases opposite, effects on cardiac function [37]. It is known that NOS1 and CaMKII are spatially coupled in the SR, with a facilitative effect on contractility $[37,38]$. Interestingly, NO has been recently demonstrated to be responsible for a novel activation scheme for CaMKII in cardiomyocytes, being the increase in NO the consequence of AKT-dependent NOS1 stimulation [27,28,39]. In this pathway, activation of CaMKII occurs likely by nitrosylation of residues within the regulatory domain of the kinase, thus allowing for its increased activity [40]. The increase in NO bioavailability would be the result not only of an AKT-dependent phosphorylation of NOS1 
A

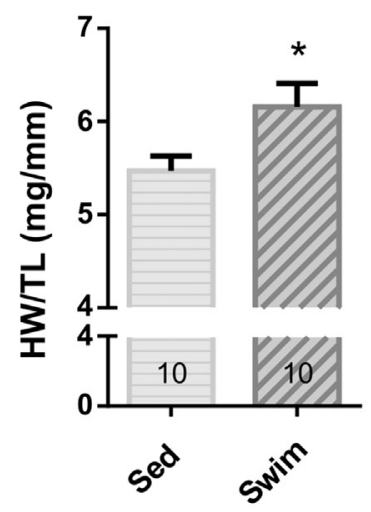

D

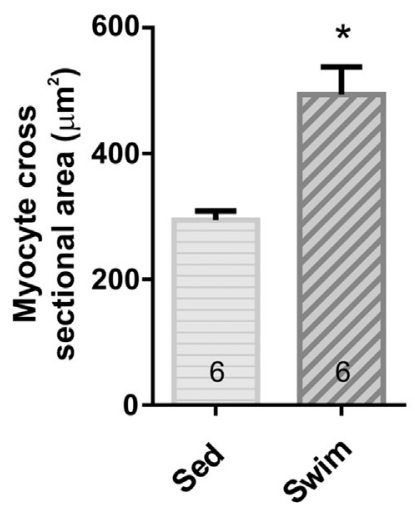

B

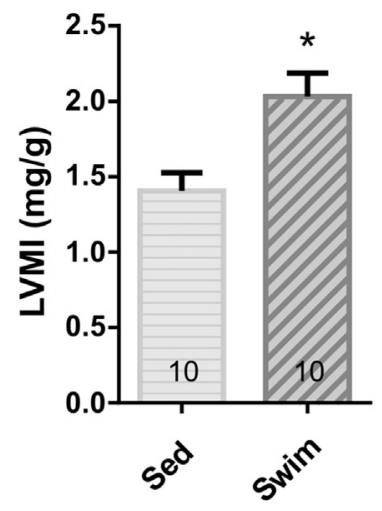

E

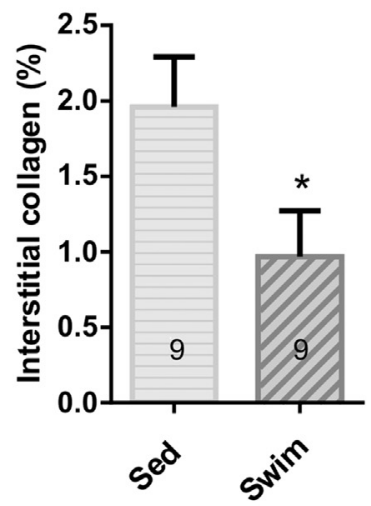

C

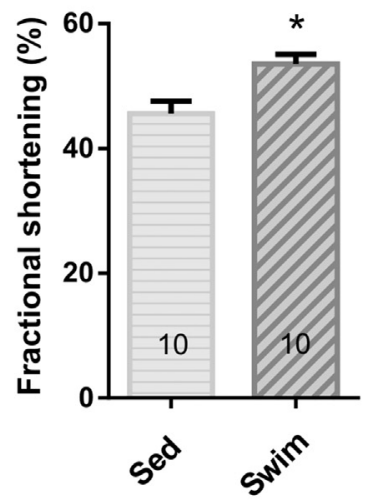

F

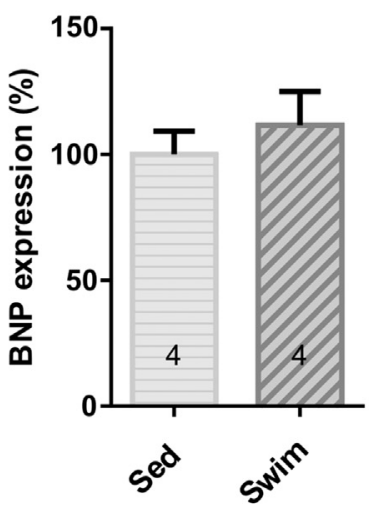

G
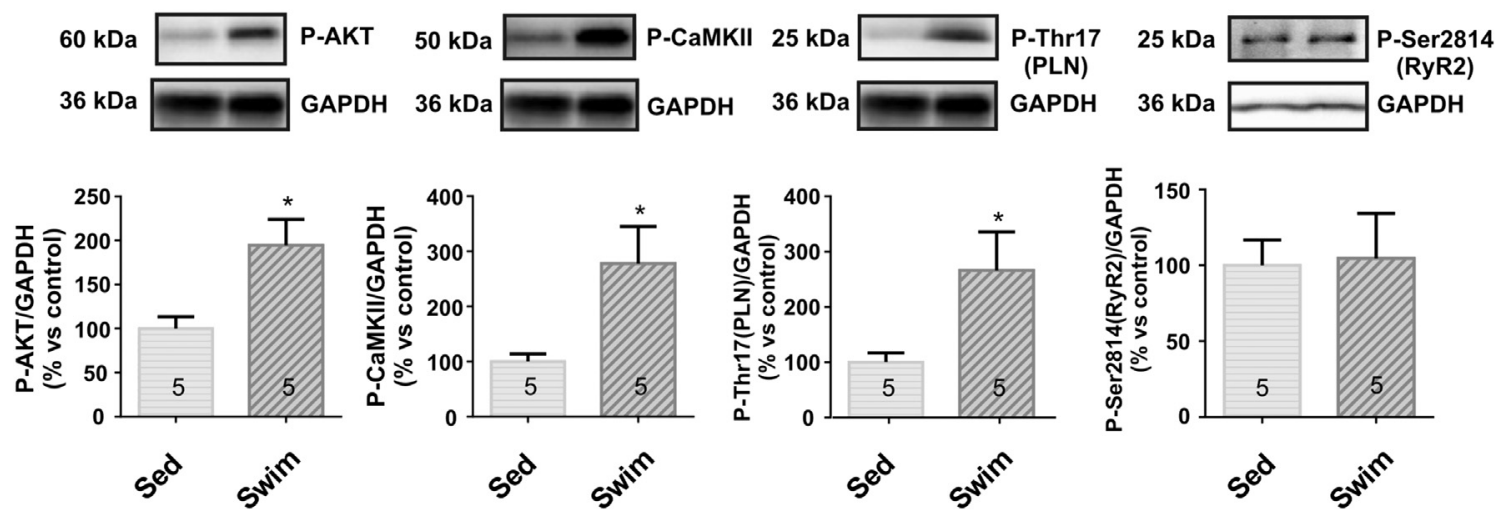

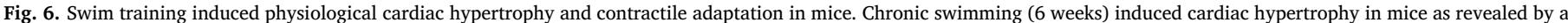

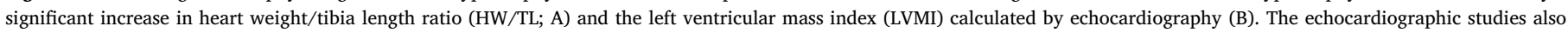

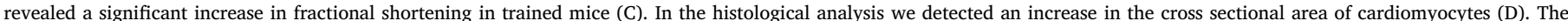

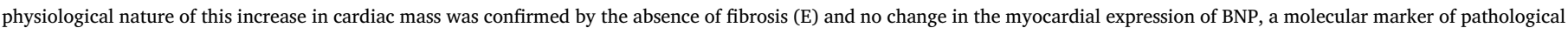

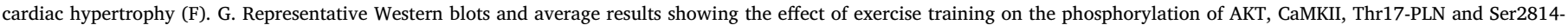
RyR2. The number of experiments $(\mathrm{n})$ in each group is depicted inside the bars. * means $\mathrm{p}<0.05$, Wilcoxon rank-sum test.

(present results) but also due to a greater expression induced by chronic training [17]. Regarding cardiomyocyte NOS3, it has been recently demonstrated that it is not stimulated by exercise training, and thus, does not to contribute to cardioprotection [41]. Our results exploring the effect of IGF-1 on cardiomyocyte cell shortening in the presence and absence NOS1 inhibition, as well as those revealing the stimulatory effect of IGF-1 upon NO production and the prevention of IGF-1 induced CaMKII activation by the NOS1 inhibitor NG, support that this pathway is critically involved in the positive inotropic response to IGF-
1. Hence, NO-induced CaMKII activation would be responsible for the increase in contractility detected in the presence of IGF-1. Interestingly, these results are in agreement with the recent demonstration of Erickson et al. [40] that NO-induced S-nitrosylation of the Cys-290 site results in persistent increase in autonomous CaMKII activity. Exploring the effect of IGF-1 upon cardiac contractility in isolated cardiomyocytes simplified the interpretation of the results by avoiding the consequences of IGF-1 on other tissues, such as the reduction in afterload due to the endothelium-dependent vasodilation. 
A

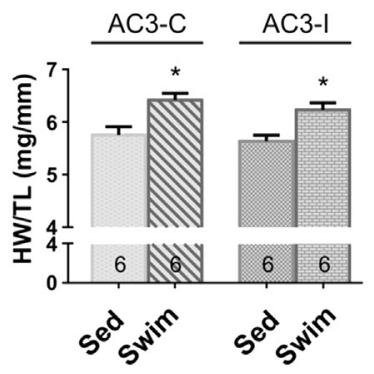

B

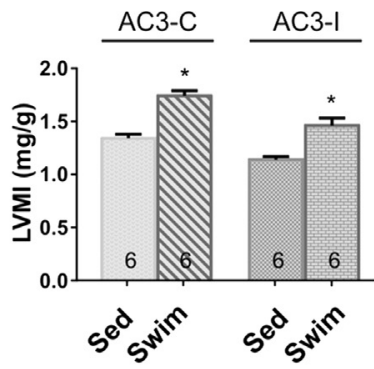

C

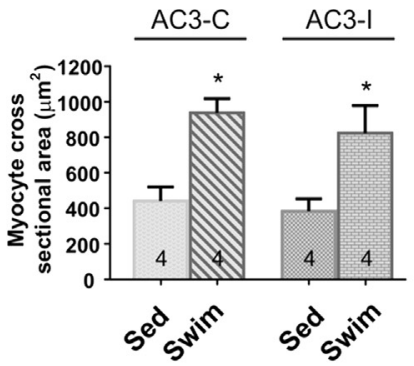

D

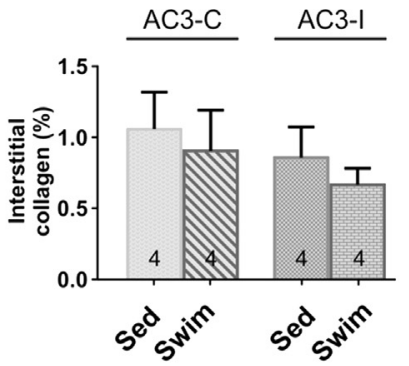

E

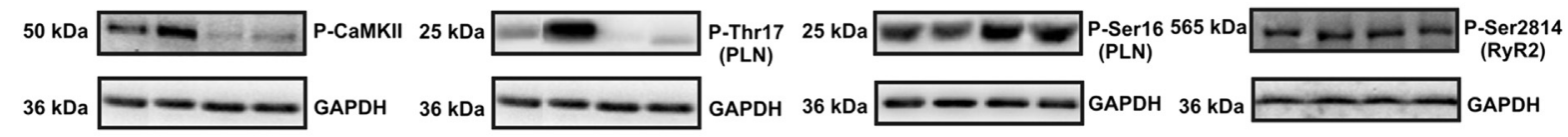

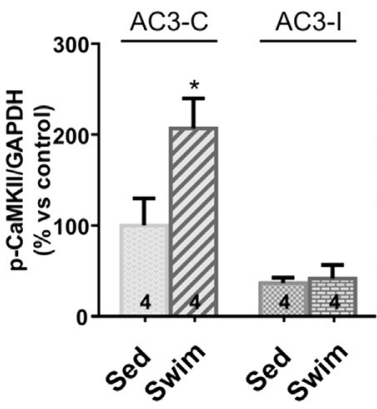

F

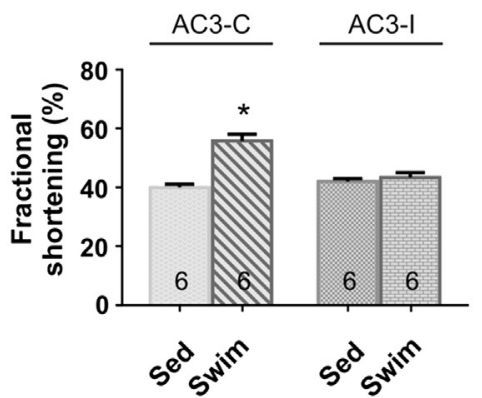

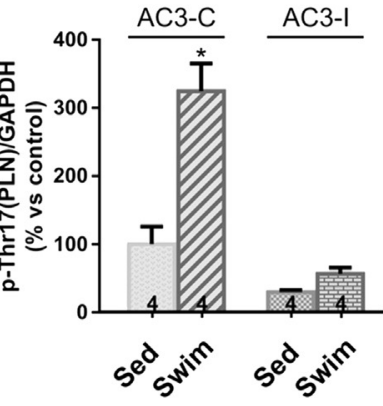

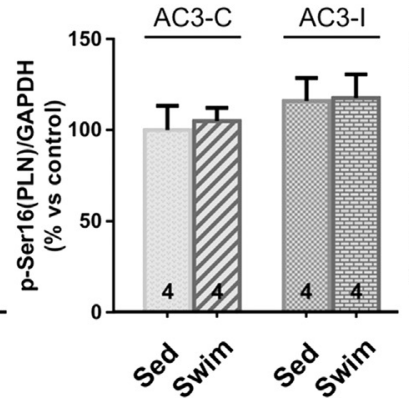

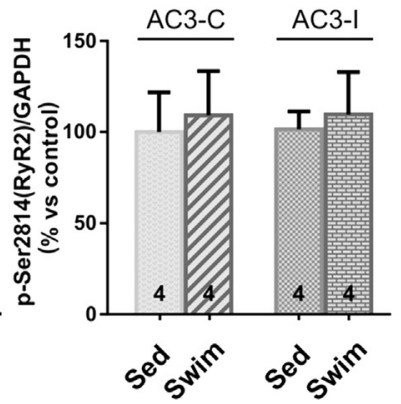

G

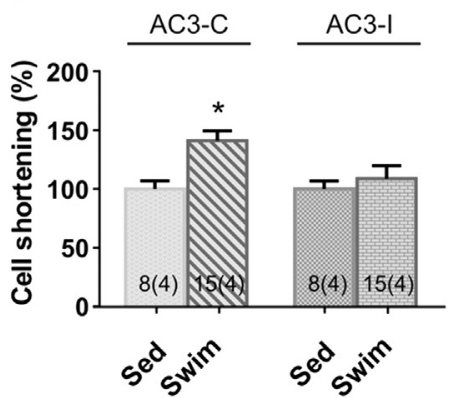

$\mathrm{H}$

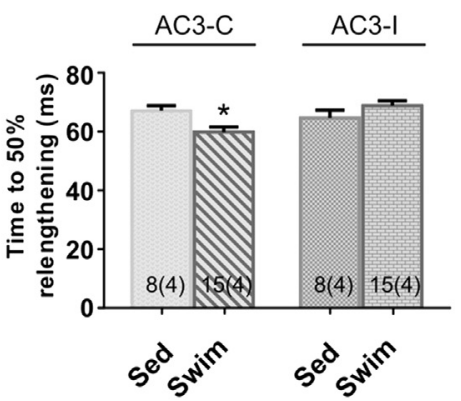

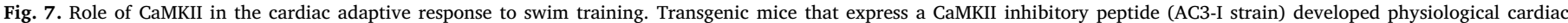

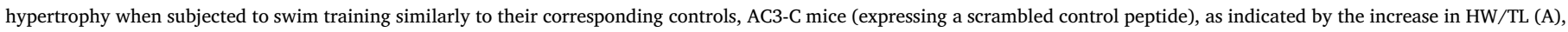

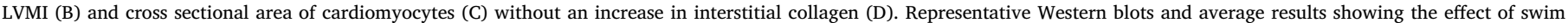

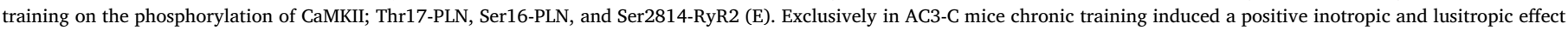

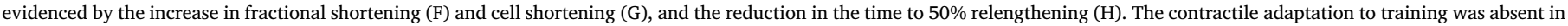

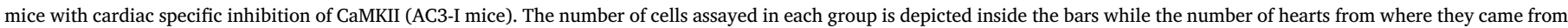
is specified between parentheses. * Indicates p $<0.05$ vs. sedentary mice for each strain, Wilcoxon rank-sum test.

The fact that CaMKII activation was mandatory for the contractile response to IGF-1, as demonstrated by the absence of the positive inotropic effect not only in the presence of the specific inhibitor of CaMKII, KN-93, but also in AC3-I transgenic mice; in combination with normal development of physiological cardiac hypertrophy induced by the swim routine in AC3-I mice, unmasked a key point of divergence in the signaling pathway that leads to either cardiac hypertrophy or to the contractile adaptation to exercise training. CaMKII activation while being critical for the inotropic response would not be required for the increase in cardiac mass in response to exercise training/IGF-1 release.

PLN, a constitutive regulator of SERCA2a function, is a well-known substrate for CaMKII. Once CaMKII is activated in the SR microdomain, in this case by the NOS1-dependent increase in NO, it phosphorylates the residue Thr17 of PLN allowing a higher activity of SERCA2a and therefore improving $\mathrm{Ca}^{2+}$ handling and contractility. Our results showing that IGF-1 increases $\mathrm{Ca}^{2+}$ transient amplitude and SR $\mathrm{Ca}^{2+}$ content -as revealed by the greater amplitude of the caffeine-induced $\mathrm{Ca}^{2+}$ transient in the presence of IGF-1- support this hypothesis and are in agreement with those of Kemi et al. [19]. These authors described an increase in CaMKII and Thr17-PLN phosphorylation together with enhanced myocardial contractility in mice subjected to endurance training during 6 weeks. The relationship between NOS1 and PLN phosphorylation is also supported by the slower rate of intracellular $\mathrm{Ca}^{2+}$ decline and relengthening in NOS1 knockout mice (NOS1 ${ }^{-/-}$) [42-44]. Interestingly, the work of Roof et al. [17,18] also support NOS1-dependent CaMKII activation during exercise training. However, 


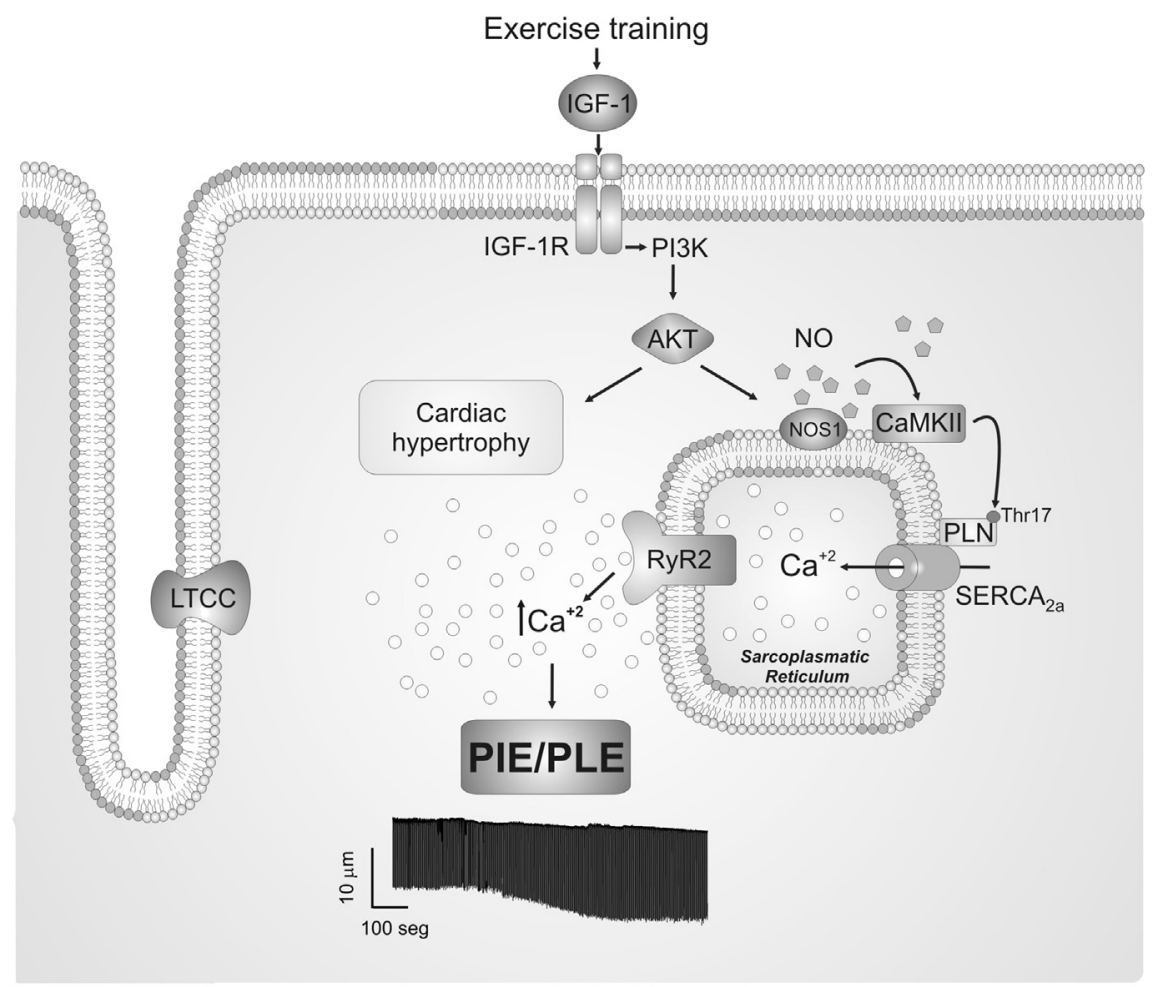

Fig. 8. Schematic summary of the molecular events proposed to underlie the adaptive cardiac contractile response to exercise training/IGF-1 release. Briefly, exercise training, through the release of IGF-1, induces the phosphorylation of AKT which in turn activates NOS1 increasing myocardial NO production. This increase in NO would be responsible for CaMKII activation that phosphorylates residue Thr17-PLN increasing SERCA2a activity and SR $\mathrm{Ca}^{2+}$ content, being the functional consequences a positive inotropic and lusitropic effect. LTCC: L-type $\mathrm{Ca}^{2+}$ channel; RyR2: ryanodine receptor 2; PIE: positive inotropic effect; PLE: positive lusitropic effect.

these authors attributed the improvement in cardiomyocyte $\mathrm{Ca}^{2+}$ handling to an increase in Ser16-PLN phosphorylation with no detectable changes in Thr17-PLN phosphorylation. Indeed, they ruled out an enhancement in PKA activity, the kinase responsible for Ser16-PLN phosphorylation, and proposed a CaMKII-dependent inhibition of phosphatases as the mechanism underling the positive inotropic effect $[17,18]$. We do not have an explanation for this discrepancy at present.

PLN is not the only target of CaMKII that can be involved in the positive inotropic effect of IGF-1. Among the other possibilities, an increase in $\mathrm{Ca}^{2+}$ current $\left(I \mathrm{Ca}_{\mathrm{L}}\right)$ through CaMKII-dependent phosphorylation of the L-type $\mathrm{Ca}^{2+}$ channel, may also enhance $\mathrm{Ca}^{2+}$ transients and contractility. Regarding this mechanism, Sun et al. [45] demonstrated that IGF-1 enhances cardiac L-type $\mathrm{Ca}^{2+}$ current through a PI3K/AKT dependent mechanism. Moreover, in the setting of chronic exercise training it was recently proposed that AKT directly controls Ltype $\mathrm{Ca}^{2+}$ channel complex protein density and $I_{\mathrm{Ca}, \mathrm{L}}$ by preventing degradation of the pore-forming subunit of the L-type $\mathrm{Ca}^{2+}$ channel complex [46]. Our results showing that pharmacological or genetic inhibition of CaMKII are able to completely abolish the IGF-1-induced positive inotropic effect suggest that if L-type $\mathrm{Ca}^{2+}$ current contributes to the acute IGF-1-induced positive inotropic effect it is through a CaMKII-dependent mechanism. Whether direct, AKT-dependent enhancement of $I \mathrm{Ca}_{\mathrm{L}}$ coexists with Thr17 of PLN in the chronic response to exercise training warrants further investigation.

Interestingly, the NOS-CaMKII pathway described herein has been shown to contribute significantly not only to the inotropic effect but also to the arrhythmogenic potential of $\beta$-AR stimulation. The latter seems to be the consequence of CaMKII-dependent phosphorylation of RyR2 favoring SR $\mathrm{Ca}^{2+}$ leak and spontaneous $\mathrm{Ca}^{2+}$ waves $[27,28]$. Since the cardiac physiologic adaptation to moderate intensity exercise training does not involve a greater susceptibility to arrhythmias [47-51] and we did not detect an increase in the phosphorylation of Ser2814-RyR2 either in IGF-1 stimulated hearts or trained mice, it is tempting to speculate that beneficial or detrimental consequence on $\mathrm{Ca}^{2+}$ handling could depend on spatial/temporal compartmentalization of NO-dependent activation of CaMKII. Thus, under physiologic conditions, i.e. exercise training; CaMKII nitrosylation improves
SERCA2a activity through the phosphorylation of Thr17-PLN. However, under pathologic circumstances, i.e. pressure overload, CaMKII would also phosphorylate RyR2 inducing SR $\mathrm{Ca}^{2+}$ leak and providing an arrhythmogenic substrate.

In summary, based on the presented data we propose that CaMKII plays an essential role in the adaptive improvement in contractility associated to exercise training, but not in the expected physiological increase in cardiac mass. Exercise training, through the release of IGF-1, would induce the sequential activation of IGF-1 receptor, AKT and NOS1. The increase in myocardial NO would activate CaMKII leading to the phosphorylation of Thr17-PLN increasing SERCA2a activity and therefore contractility. Fig. 8 schematically summarizes this proposal.

\section{Acknowledgements}

We thank Dr. Erica Pereyra, Omar Castillo, Lucia Pagola and Monica Rando for their technical assistance. We also thank Dr. Mark Anderson from Johns Hopkins University, Baltimore, MD, for the generous gift of AC3 mice breeding pairs.

\section{Funding sources}

This work was supported in part by grant PICT 2012-2907, from Agencia Nacional de Promoción Científica of Argentina and PIP 0433 from Consejo Nacional de Investigaciones Científicas y Tecnológicas (CONICET) to Dr. Irene L. Ennis and grant PICT 2014-1678, from Agencia Nacional de Promoción Científica of Argentina to Martin Vila Petroff.

\section{Disclosures}

None to declare.

\section{References}

[1] C.D. Garciarena, O.A. Pinilla, M.B. Nolly, R.P. Laguens, E.M. Escudero, H.E. Cingolani, et al., Endurance training in the spontaneously hypertensive rat: 
conversion of pathological into physiological cardiac hypertrophy, Hypertension 53 (2009) 708-714.

[2] S. Gielen, G. Schuler, V. Adams, Cardiovascular effects of exercise training: molecular mechanisms, Circulation 122 (2010) 1221-1238.

[3] R. Hambrecht, S. Gielen, A. Linke, E. Fiehn, J. Yu, C. Walther, et al., Effects of exercise training on left ventricular function and peripheral resistance in patients with chronic heart failure: a randomized trial, JAMA 283 (2000) 3095-3101.

[4] P. Giannuzzi, P.L. Temporelli, U. Corra, L. Tavazzi, Antiremodeling effect of long term exercise training in patients with stable chronic heart failure: results of the Exercise in Left Ventricular Dysfunction and Chronic Heart Failure (ELVD-CHF) Trial, Circulation 108 (2003) 554-559.

[5] M. Scheinowitz, G. Kessler-Icekson, S. Freimann, R. Zimmermann, W. Schaper, E. Golomb, et al., Short- and long-term swimming exercise training increases myocardial insulin-like growth factor-I gene expression, Growth Hormon. IGF Res. 13 (2003) 19-25.

[6] G.G. Neri Serneri, M. Boddi, P.A. Modesti, I. Cecioni, M. Coppo, L. Padeletti, et al., Increased cardiac sympathetic activity and insulin-like growth factor-I formation are associated with physiological hypertrophy in athletes, Circ. Res. 89 (2001) 977-982.

[7] R. Troncoso, C. Ibarra, J.M. Vicencio, E. Jaimovich, S. Lavandero, New insights into IGF-1 signaling in the heart, Trends Endocrinol. Metab. 25 (2014) 128-137.

[8] J. Kim, A.R. Wende, S. Sena, H.A. Theobald, J. Soto, C. Sloan, et al., Insulin-like growth factor I receptor signaling is required for exercise-induced cardiac hypertrophy, Mol. Endocrinol. 22 (2008) 2531-2543.

[9] B. DeBosch, I. Treskov, T.S. Lupu, C. Weinheimer, A. Kovacs, M. Courtois, et al., Akt 1 is required for physiological cardiac growth, Circulation 113 (2006) 2097-2104.

[10] J.R. McMullen, T. Shioi, L. Zhang, O. Tarnavski, M.C. Sherwood, P.M. Kang, et al., Phosphoinositide 3-kinase (p110alpha) plays a critical role for the induction of physiological, but not pathological, cardiac hypertrophy, Proc. Natl. Acad. Sci. U. S. A. 100 (2003) 12355-12360.

[11] J.R. McMullen, T. Shioi, W.Y. Huang, L. Zhang, O. Tarnavski, E. Bisping, et al., The insulin-like growth factor 1 receptor induces physiological heart growth via the phosphoinositide 3-kinase(p110alpha) pathway, J. Biol. Chem. 279 (2004) $4782-4793$.

[12] B.T. O'Neill, J. Kim, A.R. Wende, H.A. Theobald, J. Tuinei, J. Buchanan, et al., A conserved role for phosphatidylinositol 3-kinase but not Akt signaling in mitochondrial adaptations that accompany physiological cardiac hypertrophy, Cell Metab. 6 (2007) 294-306.

[13] S. Kinugawa, H. Tsutsui, T. Ide, R. Nakamura, K. Arimura, K. Egashira, et al., Positive inotropic effect of insulin-like growth factor-1 on normal and failing cardiac myocytes, Cardiovasc. Res. 43 (1999) 157-164.

[14] J. Ren, L. Jefferson, J.R. Sowers, R.A. Brown, Influence of age on contractile response to insulin-like growth factor 1 in ventricular myocytes from spontaneously hypertensive rats, Hypertension 34 (1999) 1215-1222.

[15] J. Ren, M.F. Walsh, M. Hamaty, J.R. Sowers, R.A. Brown, Altered inotropic response to IGF-I in diabetic rat heart: influence of intracellular Ca2 + and NO, Am. J. Phys. 275 (1998) H823-30.

[16] L. Yang, Z. Jia, M. Zhu, J. Zhang, J. Liu, P. Wu, et al., Exercise protects against chronic beta-adrenergic remodeling of the heart by activation of endothelial nitric oxide synthase, PLoS One 9 (2014) e96892.

[17] S.R. Roof, L. Tang, J.E. Ostler, M. Periasamy, S. Gyorke, G.E. Billman, et al., Neuronal nitric oxide synthase is indispensable for the cardiac adaptive effects of exercise, Basic Res. Cardiol. 108 (2013) 332.

[18] S.R. Roof, H.T. Ho, S.C. Little, J.E. Ostler, E.A. Brundage, M. Periasamy, et al., Obligatory role of neuronal nitric oxide synthase in the heart's antioxidant adaptation with exercise, J. Mol. Cell. Cardiol. 81 (2015) 54-61.

[19] O.J. Kemi, O. Ellingsen, M. Ceci, S. Grimaldi, G.L. Smith, G. Condorelli, et al., Aerobic interval training enhances cardiomyocyte contractility and $\mathrm{Ca} 2+$ cycling by phosphorylation of CaMKII and Thr-17 of phospholamban, J. Mol. Cell. Cardiol. 43 (2007) 354-361.

[20] G. Kaurstad, M.N. Alves, O.J. Kemi, N. Rolim, M.A. Hoydal, H. Wisloff, et al., Chronic CaMKII inhibition blunts the cardiac contractile response to exercise training, Eur. J. Appl. Physiol. 112 (2012) 579-588.

[21] O.J. Kemi, M. Ceci, G. Condorelli, G.L. Smith, U. Wisloff, Myocardial sarcoplasmic reticulum $\mathrm{Ca} 2$ + ATPase function is increased by aerobic interval training, Eur. J. Cardiovasc. Prev. Rehabil. 15 (2008) 145-148.

[22] N.P. Rolim, A. Medeiros, K.T. Rosa, K.C. Mattos, M.C. Irigoyen, E.M. Krieger, et al., Exercise training improves the net balance of cardiac Ca2 + handling protein expression in heart failure, Physiol. Genomics 29 (2007) 246-252.

[23] L. Lu, D.F. Mei, Gu AG, S. Wang, B. Lentzner, D.E. Gutstein, et al., Exercise training normalizes altered calcium-handling proteins during development of heart failure, J. Appl. Physiol. (1985) 92 (2002) 1524-1530.

[24] S.J. Kim, M. Abdellatif, S. Koul, G.J. Crystal, Chronic treatment with insulin-like growth factor I enhances myocyte contraction by upregulation of Akt-SERCA2a signaling pathway, Am. J. Physiol. Heart Circ. Physiol. 295 (2008) H130-5.

[25] Kawasaki K, Smith RS, Jr., Hsieh CM, Sun J, Chao J, Liao JK. Activation of the phosphatidylinositol 3-kinase/protein kinase Akt pathway mediates nitric oxideinduced endothelial cell migration and angiogenesis. Mol. Cell. Biol. 2003; 23:5726-37.

[26] M.P. Haynes, D. Sinha, K.S. Russell, M. Collinge, D. Fulton, M. Morales-Ruiz, et al.,
Membrane estrogen receptor engagement activates endothelial nitric oxide synthase via the PI3-kinase-Akt pathway in human endothelial cells, Circ. Res. 87 (2000) 677-682.

[27] J. Curran, L. Tang, S.R. Roof, S. Velmurugan, A. Millard, S. Shonts, et al., Nitric oxide-dependent activation of CaMKII increases diastolic sarcoplasmic reticulum calcium release in cardiac myocytes in response to adrenergic stimulation, PLoS One 9 (2014) e87495.

[28] D.A. Gutierrez, M. Fernandez-Tenorio, J. Ogrodnik, E. Niggli, NO-dependent CaMKII activation during beta-adrenergic stimulation of cardiac muscle, Cardiovasc. Res. 100 (2013) 392-401.

[29] R. Zhang, M.S. Khoo, Y. Wu, Y. Yang, C.E. Grueter, G. Ni, et al., Calmodulin kinase II inhibition protects against structural heart disease, Nat. Med. 11 (2005) 409-417.

[30] M.L. Schuman, M.S. Landa, J.E. Toblli, L.S. Peres Diaz, A.L. Alvarez, S. Finkielman, et al., Cardiac thyrotropin-releasing hormone mediates left ventricular hypertrophy in spontaneously hypertensive rats, Hypertension 57 (2011) 103-109.

[31] X. Wang, T. Oka, F.L. Chow, S.B. Cooper, J. Odenbach, G.D. Lopaschuk, et al., Tumor necrosis factor-alpha-converting enzyme is a key regulator of agonist-induced cardiac hypertrophy and fibrosis, Hypertension 54 (2009) 575-582.

[32] J.R. Bell, C.L. Curl, T.W. Harding, M. Vila Petroff, S.B. Harrap, L.M. Delbridge, Male and female hypertrophic rat cardiac myocyte functional responses to ischemic stress and beta-adrenergic challenge are different, Biol. Sex Differ. 7 (2016) 32.

[33] J.R. Bell, A.J. Raaijmakers, C.L. Curl, M.E. Reichelt, T.W. Harding, A. Bei, et al., Cardiac CaMKIIdelta splice variants exhibit target signaling specificity and confer sex-selective arrhythmogenic actions in the ischemic-reperfused heart, Int. J. Cardiol. 181 (2015) 288-296.

[34] M. Sepulveda, L.A. Gonano, T.G. Back, S.R. Chen, M. Vila Petroff, Role of CaMKII and ROS in rapid pacing-induced apoptosis, J. Mol. Cell. Cardiol. 63 (2013) $135-145$.

[35] L.A. Gonano, M. Morell, J.I. Burgos, R.A. Dulce, V.C. De Giusti, E.A. Aiello, et al., Hypotonic swelling promotes nitric oxide release in cardiac ventricular myocytes: impact on swelling-induced negative inotropic effect, Cardiovasc. Res. 104 (2014) $456-466$.

[36] A.M. Yeves, M.C. Villa-Abrille, N.G. Perez, A.J. Medina, E.M. Escudero, I.L. Ennis, Physiological cardiac hypertrophy: critical role of AKT in the prevention of NHE-1 hyperactivity, J. Mol. Cell. Cardiol. 76C (2014) 186-195.

[37] L.A. Barouch, R.W. Harrison, M.W. Skaf, G.O. Rosas, T.P. Cappola, Z.A. Kobeissi, et al., Nitric oxide regulates the heart by spatial confinement of nitric oxide synthase isoforms, Nature 416 (2002) 337-339.

[38] Z. Jian, H. Han, T. Zhang, J. Puglisi, L.T. Izu, J.A. Shaw, et al., Mechanochemotransduction during cardiomyocyte contraction is mediated by localized nitric oxide signaling, Sci. Signal. 7 (2014) ra27.

[39] L. Pereira, D.J. Bare, S. Galice, T.R. Shannon, D.M. Bers, beta-Adrenergic induced SR Ca2 + leak is mediated by an Epac-NOS pathway, J. Mol. Cell. Cardiol. 108 (2017) 8-16.

[40] J.R. Erickson, C.B. Nichols, H. Uchinoumi, M.L. Stein, J. Bossuyt, D.M. Bers, SNitrosylation induces both autonomous activation and inhibition of calcium/calmodulin-dependent protein kinase II delta, J. Biol. Chem. 290 (2015) 25646-25656.

[41] C. Farah, A. Nascimento, G. Bolea, G. Meyer, S. Gayrard, A. Lacampagne, et al., Key role of endothelium in the eNOS-dependent cardioprotection with exercise training, J. Mol. Cell. Cardiol. 102 (2016) 26-30.

[42] E.A. Ashley, C.E. Sears, S.M. Bryant, H.C. Watkins, B. Casadei, Cardiac nitric oxide synthase 1 regulates basal and beta-adrenergic contractility in murine ventricular myocytes, Circulation 105 (2002) 3011-3016.

[43] S.A. Khan, M.W. Skaf, R.W. Harrison, K. Lee, K.M. Minhas, A. Kumar, et al., Nitric oxide regulation of myocardial contractility and calcium cycling: independent impact of neuronal and endothelial nitric oxide synthases, Circ. Res. 92 (2003) 1322-1329.

[44] C.E. Sears, S.M. Bryant, E.A. Ashley, C.A. Lygate, S. Rakovic, H.L. Wallis, et al., Cardiac neuronal nitric oxide synthase isoform regulates myocardial contraction and calcium handling, Circ. Res. 92 (2003) e52-9.

[45] H. Sun, B.G. Kerfant, D. Zhao, M.G. Trivieri, G.Y. Oudit, J.M. Penninger, et al., Insulin-like growth factor-1 and PTEN deletion enhance cardiac L-type Ca2 + currents via increased PI3Kalpha/PKB signaling, Circ. Res. 98 (2006) 1390-1397.

[46] D. Catalucci, D.H. Zhang, J. DeSantiago, F. Aimond, G. Barbara, J. Chemin, et al., Akt regulates L-type Ca2 + channel activity by modulating Cavalpha1 protein stability, J. Cell Biol. 184 (2009) 923-933.

[47] K.C. Yang, N.C. Foeger, C. Marionneau, P.Y. Jay, J.R. McMullen, J.M. Nerbonne, Homeostatic regulation of electrical excitability in physiological cardiac hypertrophy, J. Physiol. 588 (2010) 5015-5032.

[48] M. Halle, M. Huonker, S.H. Hohnloser, M. Alivertis, A. Berg, J. Keul, QT dispersion in exercise-induced myocardial hypertrophy, Am. Heart J. 138 (1999) 309-312.

[49] T.M. Eijsvogels, A.B. Fernandez, P.D. Thompson, Are there deleterious cardiac effects of acute and chronic endurance exercise? Physiol. Rev, 96 (2016) 99-125.

[50] N. Rolim, K. Skardal, M. Hoydal, M.M. Sousa, V. Malmo, G. Kaurstad, et al., Aerobic interval training reduces inducible ventricular arrhythmias in diabetic mice after myocardial infarction, Basic Res. Cardiol. 110 (2015) 44.

[51] A. Biffi, B.J. Maron, B. Di Giacinto, P. Porcacchia, L. Verdile, F. Fernando, et al., Relation between training-induced left ventricular hypertrophy and risk for ventricular tachyarrhythmias in elite athletes, Am. J. Cardiol. 101 (2008) 1792-1795. 